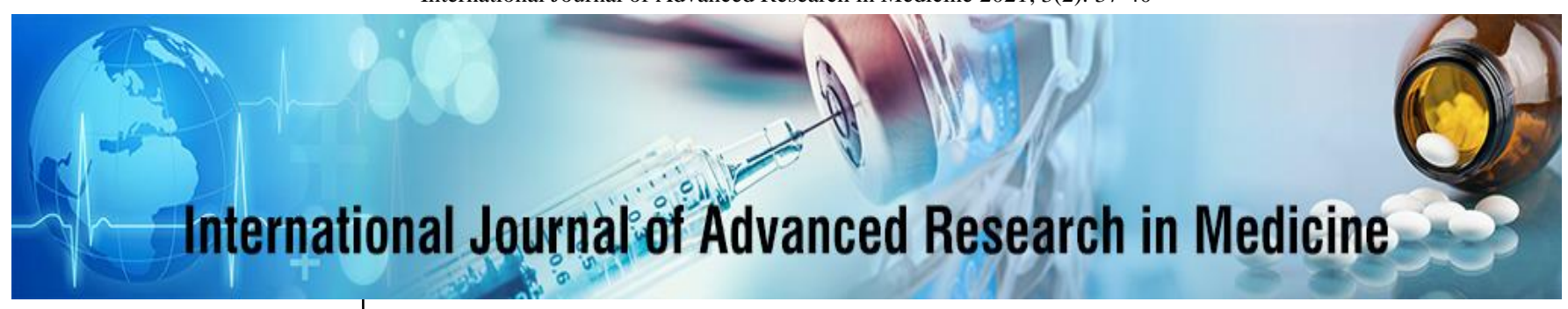

E-ISSN: 2706-9575

P-ISSN: 2706-9567

IJARM 2021; 3(2): 37-40

Received: 05-05-2021

Accepted: 17-06-2021

Dr. Kavangal Dhananjay Assistant Professor, Department of General Medicine, Bhaskara Medical College, Moinabad,

Rangareddy, Telangana, India

Dr. Mamatha Pulloori Assistant Professor, Department of General Medicine, MNR Medical College and Hospital, Sangareddy, Telangana, India

Corresponding Author: Dr. Mamatha Pulloori Assistant Professor, Department of General Medicine, MNR Medical College and Hospital, Sangareddy, Telangana, India

\section{Aspartate aminotransferase platelet ratio index (APRI) and fibroscan in predicting early fibrosis in non-alcoholic fatty liver diseases cases}

\author{
Dr. Kavangal Dhananjay and Dr. Mamatha Pulloori
}

DOI: https://doi.org/10.22271/27069567.2021.v3.i2a.215

\begin{abstract}
Nonalcoholic fatty liver disease (NAFLD) is emerging as an important cause of chronic liver disease in worldwide. Liver biopsy is a gold standard procedure in the diagnosis and staging of NAFLD, but there are several non-invasive diagnostic procedures have been emerged for the early diagnosis of liver fibrosis. The present study was designed to assess the efficacy of APRI to fibroscan as a marker for early fibrosis in cases with non-alcoholic fatty liver diseases. A total of 120 cases, which are newly diagnosed with non-alcoholic fatty liver disease attending OPD were recruited. Laboratory investigation results were obtained after overnight for evaluation of complete blood count, blood glucose levels, albumin, total bilirubin, platelet count, prothrombin time international normalized ratio, cholesterol, triglyceride (TG), serum urea, creatinine, HBsAg, HCV. Radiological evaluation was conducted by USG abdomen and fibroscan. The value of triglyceride was below $150 \mathrm{mg} / \mathrm{dl}$ in $34.16 \%$ cases, between $150-200 \mathrm{mg} / \mathrm{dl}$ in $45.83 \%$ cases and $>200 \mathrm{mg} / \mathrm{dl}$ in $20 \%$ cases. The fasting blood sugar was $>100$ in $40 \%$ cases and $<100$ in $60 \%$ cases. The aspartate aminotransferase levels was $<30 \mathrm{IU} / \mathrm{L}$ in $17.5 \%$ cases, $31-40$ IU/L in $29.16 \%, 41-50$ IU/L in $33.33 \%$, and $>50 \mathrm{IU} / \mathrm{L}$ in $20 \%$ cases. The platelets count was $<100$ in $29.16 \%$ cases, $101-150$ in $53.33 \%$ cases, $151-200$ in $12.5 \%$ cases and $>200$ in $5 \%$ cases. The fibroscan values was $<7.0$ in $45 \%$ cases, $7.1-8.9$ in $35 \%$ cases and $>9.0$ in $20 \%$ cases. The APRI score was $<0.7$ in $33.3 \%$ cases, $0.7-1.0$ in $26.66 \%$ cases and $>1.0$ in $40 \%$ cases. There is a significant correlation between fibroscan and APRI in cases with NAFLD. Fibroscan and APRI are uniformly efficient in predicting liver fibrosis. The use of APRI $>0.7$ would avoid the need for fibroscan.
\end{abstract}

Keywords: nonalcoholic fatty liver disease (NAFLD), aminotransferase platelet ratio index (APRI), liver fibrosis

\section{Introduction}

Liver disease represents a rapidly increasing health burden globally. The prevalence of NAFLD is around 9-32\% of general population in India. Clinico-pathological studies show that NAFLD is an important cause of unexplained rise in hepatic transaminases, cryptogenic cirrhosis and cryptogenic hepatocellular carcinoma in Indian patients ${ }^{[1]}$. The invention of new diagnostic noninvasive markers of nonalcoholic fatty liver disease (NAFLD) is a major preference in clinical research. Analyses of liver parameters are now the third most common type of biochemical test ${ }^{[2,3]}$.

The transaminases, alanine aminotransferase (ALT) and aspartate aminotransferase (AST), which indicate the damage of hepatic cells and are most used liver test in clinical practice. Raise in ALT and AST levels has been used as indicators for further liver investigations. The recent guidelines of United Kingdom concluded that there is no evidence for this strategy ${ }^{[4]}$. Elevated transaminases are a common finding affecting around $10 \%$ of the general population, while only a minority of these persons will develop liver-related events ${ }^{[5-7]}$. Liver biopsy has been considered as gold standard technique in the diagnosis of liver fibrosis. Liver biopsy technique is non-invasive procedure with limitations and $0.05 \%$ mortality. With this there is immediate need of non-invasive procedure like aminotransferase platelet ratio index (APRI) and fibroscan in the early diagnosis of liver fibrosis. Fibroscan has good sensitivity, specificity and diagnostic accuracy. The present study was designed to assess the efficacy of APRI to fibroscan as a marker for early fibrosis in cases with nonalcoholic fatty liver diseases. 


\section{Material and Methods}

The present prospective study was conducted in the Department of General Medicine at MNR Medical College and Hospital, Sangareddy and Bhaskara medical College, Moinabad from July 2020 to March 2021. A total of 120 cases, which are newly diagnosed with non-alcoholic fatty liver disease attending OPD were recruited. Cases above 21 years of age, newly diagnosed patients of USG defined nonalcoholic fatty liver disease and cases willing to participate in the study were included. Cases with cardiovascular diseases, chronic kidney disease, chronic liver disease, consuming alcohol more than $20 \mathrm{gm} /$ day, carcinoma liver and cases not willing to participate in the study were excluded. Informed consent was obtained from all the study participants and study protocol was approved by institutional ethics committee.
All the study participants were subjected to detailed clinical, laboratory and radiological examination. Height, weight and BMI were measured. Laboratory investigation results were obtained after overnight for evaluation of complete blood count, blood glucose levels, albumin, total bilirubin, platelet count, prothrombin time international normalized ratio, cholesterol, triglyceride (TG), serum urea, creatinine, $\mathrm{HBsAg}, \mathrm{HCV}$. Radiological evaluation was conducted by USG abdomen and fibroscan.

The SPSS version 23 was used to carry out statistical analysis relevant to the study. The frequency and percentages (\%) were calculated. The Student ' $t$ ' test and chi-square test was used to test the significance of raw data and qualitative data. P-value of $<0.05$ was considered as statistically significant.

\section{Results}

Table 1: Demographic variables in the study participants

\begin{tabular}{|c|c|c|c|c|}
\hline \multirow{2}{*}{ Demographic variable } & \multicolumn{2}{|c|}{ Cases } & \multicolumn{2}{|c|}{ Controls } \\
\hline & Frequency & Percentage & Frequency & Percentage \\
\hline \multicolumn{5}{|c|}{ Age $($ In years) } \\
\hline $21-30$ & 01 & $0.83 \%$ & 01 & $0.83 \%$ \\
\hline $31-40$ & 14 & $11.66 \%$ & 15 & $12.5 \%$ \\
\hline $41-50$ & 68 & $56.67 \%$ & 80 & $66.67 \%$ \\
\hline $51-60$ & 25 & $20.83 \%$ & 14 & $11.67 \%$ \\
\hline Above 60 & 12 & $10 \%$ & 10 & $8.33 \%$ \\
\hline \multicolumn{5}{|c|}{ Gender } \\
\hline Male & 72 & $60 \%$ & 85 & $70.83 \%$ \\
\hline Female & 48 & $40 \%$ & 35 & $29.16 \%$ \\
\hline \multicolumn{5}{|c|}{ BMI } \\
\hline Lean $(<25)$ & 11 & $9.16 \%$ & 32 & $26.66 \%$ \\
\hline Overweight (26-30) & 58 & $48.33 \%$ & 68 & $56.66 \%$ \\
\hline Obese $(>30)$ & 51 & $42.5 \%$ & 20 & $16.67 \%$ \\
\hline Height $($ Mean \pm SD $)$ & \multicolumn{2}{|c|}{$159.8 \pm 10.6$} & \multicolumn{2}{|c|}{$158.5 \pm 10.8$} \\
\hline Weight (Mean \pm SD) & \multicolumn{2}{|c|}{$68.3 \pm 11.24$} & \multicolumn{2}{|c|}{$70.6 \pm 12.5$} \\
\hline
\end{tabular}

Table 2: Details of laboratory investigations in the study participants

\begin{tabular}{|c|c|c|}
\hline Parameter & Frequency & Percentage \\
\hline \multicolumn{3}{|c|}{ Triglyceride (mg/dl) } \\
\hline Below 150 & 41 & $34.16 \%$ \\
\hline $150-200$ & 55 & $45.83 \%$ \\
\hline Above 200 & 24 & $20 \%$ \\
\hline \multicolumn{3}{|c|}{ Fasting blood sugar (mg/dl) } \\
\hline Above 100 & 48 & $40 \%$ \\
\hline Below 100 & 72 & $60 \%$ \\
\hline \multicolumn{3}{|c|}{ Aspartate aminotransferase (AST) (IU/L) } \\
\hline$<30$ & 21 & $17.5 \%$ \\
\hline $31-40$ & 35 & $29.16 \%$ \\
\hline $41-50$ & 40 & $33.33 \%$ \\
\hline$>50$ & 24 & $20 \%$ \\
\hline \multicolumn{3}{|c|}{ Platelet count $\left(10^{3} / \mu \mathrm{L}\right)$} \\
\hline$<100$ & 35 & $29.16 \%$ \\
\hline $101-150$ & 64 & $53.33 \%$ \\
\hline $151-200$ & 15 & $12.5 \%$ \\
\hline$>200$ & 06 & $5 \%$ \\
\hline \multicolumn{3}{|c|}{ APRI } \\
\hline$<0.7$ & 40 & $33.3 \%$ \\
\hline $0.7-1.0$ & 32 & $26.66 \%$ \\
\hline$>1.0$ & 48 & $40 \%$ \\
\hline \multicolumn{3}{|c|}{ Fibroscan } \\
\hline$<7.0$ & 54 & $45 \%$ \\
\hline $7.1-8.9$ & 42 & $35 \%$ \\
\hline$>9.0$ & 24 & $20 \%$ \\
\hline
\end{tabular}




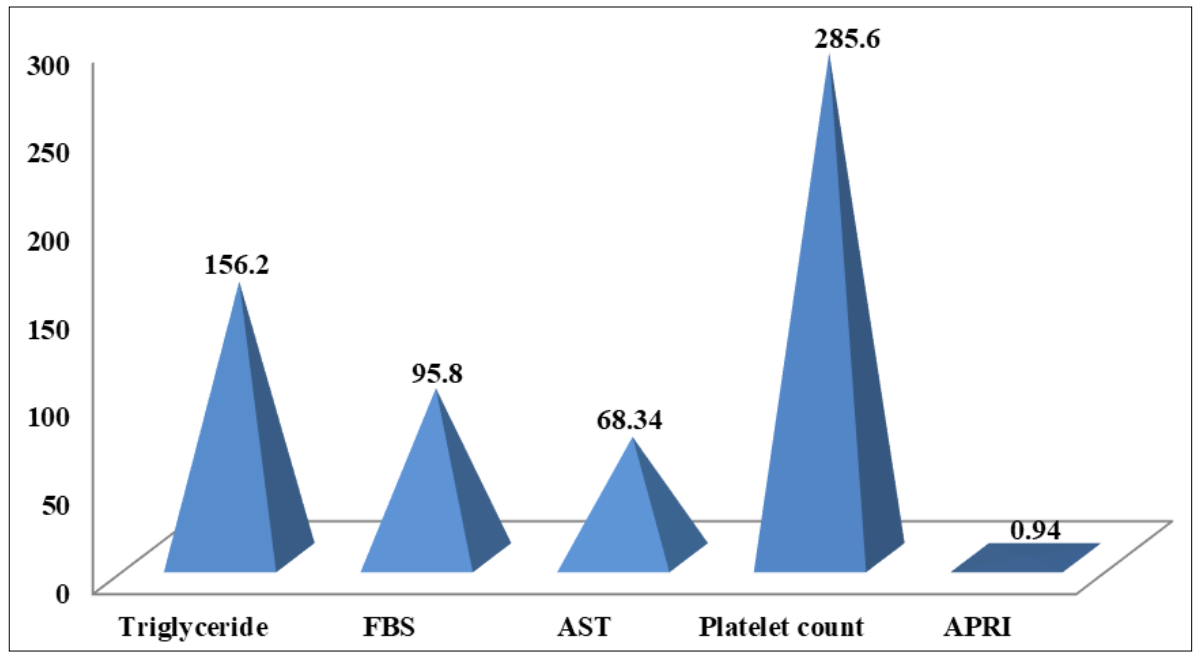

Fig 1: Mean values of laboratory investigations

Table 3: Correlation and association between fibroscan and APRI among study participants

\begin{tabular}{|c|c|c|c|}
\hline \multirow{2}{*}{ APRI } & \multicolumn{3}{|c|}{ Fibroscan } \\
\cline { 2 - 4 } & $<\mathbf{7 . 0}(\mathbf{n = 5 4})$ & $\mathbf{7 . 0 - 8 . 9}(\mathbf{n}=\mathbf{4 2})$ & $>\mathbf{9}(\mathbf{n = 2 4})$ \\
\hline$<0.7(\mathrm{n}=40)$ & 39 & 01 & 00 \\
\hline $0.7-1.0(\mathrm{n}=32)$ & 14 & 18 & 00 \\
\hline$>1.0(\mathrm{n}=48)$ & 01 & 23 & 24 \\
\hline $\mathrm{p}$-value & \multicolumn{3}{|c|}{0.0264} \\
\hline Chi square value & \multicolumn{3}{|c|}{0.854} \\
\hline Pearson correlation & \multicolumn{3}{|c}{} \\
\hline
\end{tabular}

\section{Discussion}

A total of 120 cases, which are newly diagnosed with nonalcoholic fatty liver disease attending OPD were recruited. Among the cases majority were belong to 41-50 years (56.67\%), followed by 51-60 years (20.83\%), 31-40 years $(11.66 \%)$ and above 60 years $(10 \%)$. Male participants $(60 \%)$ were more than female participants $(40 \%)$. The levels of BMI was $>30$ in $42.5 \%$ cases, in between $26-30$ in $48.33 \%$ cases and $<25$ in $9.16 \%$ cases. The mean height was $159.6 \mathrm{~cm}$ and mean weight was $68.3 \mathrm{kgs}$ (Table 1). A study by Habis YZ., noticed that an age of greater than 40 years was associated with significantly higher fibrosis compared with age 40 years or younger. There was a significant correlation between age and Fibroscan fibrosis score ${ }^{[8]}$.

The value of triglyceride was below $150 \mathrm{mg} / \mathrm{dl}$ in $34.16 \%$ cases, between $150-200 \mathrm{mg} / \mathrm{dl}$ in $45.83 \%$ cases and $>200 \mathrm{mg} / \mathrm{dl}$ in $20 \%$ cases. The fasting blood sugar was $>100$ in $40 \%$ cases and $<100$ in $60 \%$ cases. The aspartate aminotransferase levels was $<30 \mathrm{IU} / \mathrm{L}$ in $17.5 \%$ cases, $31-40$ IU/L in $29.16 \%, 41-50 \mathrm{IU} / \mathrm{L}$ in $33.33 \%$, and $>50 \mathrm{IU} / \mathrm{L}$ in $20 \%$ cases. The platelets count was $<100$ in $29.16 \%$ cases, $101-150$ in $53.33 \%$ cases, $151-200$ in $12.5 \%$ cases and $>200$ in $5 \%$ cases.

The fibroscan values was $<7.0$ in $45 \%$ cases, $7.1-8.9$ in $35 \%$ cases and $>9.0$ in $20 \%$ cases. The APRI score was $<0.7$ in $33.3 \%$ cases, $0.7-1.0$ in $26.66 \%$ cases and $>1.0$ in $40 \%$ cases. The association between APRI score and fibroscan was statistically significant $(\mathrm{p}=0.0264)$ and there was good correlation between fibroscan and APRI. A study by Habis YZ., noticed significant correlation between stiff ness score and APRI (r=0.6 and 0.65 for Pearson's and Spearman's correlation, respectively; $P<0.001$ for both. This correlation was stronger for patients who had moderate or advanced fibrosis on fibroscan ${ }^{[8]}$. The APRI cutoff of $\geq 0.7$ has a sensitivity of $77 \%$ and specificity of $72 \%$ to detect significant hepatic fibrosis and a cutoff score of 1.0 has a sensitivity of 61 to $76 \%$ and specificity of 64 to $72 \%$ for detection of severe fibrosis/cirrhosis [9]. Jayanta Paul., Stated that APRI has a good negative predictive value to exclude advanced fibrosis but does not accurately differentiate intermediate fibrosis from mild or severe fibrosis ${ }^{[10]}$. Abd El Rihim et al. reported in a meta-analysis that fibroScan had a sensitivity of $83.4 \%$ and a specificity of 92.2 for the detection of F4 fibrosis, whereas APRI had a sensitivity of $66.6 \%$ and a specificity of $71.1 \%$. However, APRI was not useful for the detection of early stages of fibrosis ${ }^{[11]}$.

A study by Habis YZ., concluded that APRI could effectively distinguish mild fibrosis and was significantly correlated with fibroscan in the assessment of severe and moderate fibrosis. The combine used of APRI and fibroscan increases the diagnostic accuracy in assessment of the stages of liver fibrosis ${ }^{[8]}$. A meta-analysis by Lin $\mathrm{ZH}$ et al. concluded that APRI can identify hepatitis C-related fibrosis with a moderate degree of accuracy. Application of this index may decrease the need for staging liver biopsy specimens ${ }^{[9]}$. A study by Jayant paul stated that multiple non-invasive tests are available for diagnosis of NAFLD, and its different stages however gold standard test is liver biopsy ${ }^{[10]}$. A study by Pathik $\mathrm{P}$ et al. concluded that fibroscan, NFS and AAR are simple non-invasive markers of fibrosis that can be utilized as screening tools in patients with high risk for fibrosis to determine the need for biopsy. The cut off of Fibroscan for stage $3 / 4$ fibrosis was $12 \mathrm{kPa}$ [12].

\section{Conclusion}

Evaluation of the status of liver fibrosis is important in the early diagnosis and management of cirrhosis in cases with NAFLD and prevention of disease progression too. The 
study results indicate that there is a significant correlation between fibroscan and APRI in cases with NAFLD. Fibroscan and APRI are uniformly efficient in predicting liver fibrosis. The use of APRI $>0.7$ would avoid the need for fibroscan.

\section{References}

1. Duseja A. Nonalcoholic fatty liver disease in India: A lot done, yet more required! Indian J Gastroenterol 2010;29(6):217-25. Doi: 10.1007/s12664-010-0069-1. Epub 2010 Dec 30. PMID: 21191681.

2. Guha IN, Parkes J, Roderick PR, Harris S, Rosenberg WM. Noninvasive markers associated with liver fibrosis in non-alcoholic fatty liver disease. Gut 2006;55:1650-60.

3. Wieckowska A, McCullough AJ, Feldstein AE. Noninvasive diagnosis and monitoring of nonalcoholic steatohepatitis: Present and future. Hepatology 2007;46:582-9.

4. Newsome PN, Cramb R, Davison SM, Dillon JF, Foulerton M, Godfrey EM et al. Guidelines on the management of abnormal liver blood tests. Gut 2018;67:6-19.

5. Ioannou GN, Boyko EJ, Lee SP. The prevalence and predictors of elevated serum aminotransferase activity in the United States in 1999-2002. Am J Gastroenterol 2006;101:76-82.

6. Caballeria L, Pera G, Arteaga I, Rodriguez L, Aluma A, Morillas RM et al. High prevalence of liver fibrosis among European adults with unknown liver disease: A population-based study. Clin Gastroenterol Hepatol 2018;16:1138-1145.e5.

7. Donnan PT, McLernon D, Dillon JF, Ryder S, Roderick $\mathrm{P}$, Sullivan F et al. Development of a decision support tool for primary care management of patients with abnormal liver function tests without clinically apparent liver disease: A record-linkage population cohort study and decision analysis (ALFIE). Health Technol Assess 2009;13(3-4):9-11, 1-134.

8. Habis YZ. Assessment of Hepatic Fibrosis Using NonInvasive Aspartate Aminotransferase to Platelets Ratio Index Compared to Hepatic Stiffness Measurements Using Transient Elastography FibroScan®. Saudi Journal of Internal Medicine 2019;9(2):27-36. https://doi.org/10.32790/sjim.2019.9.2.4

9. Lin ZH, Xin YN, Dong QJ et al. Performance of the aspartate aminotransferase-to-platelet ratio index for the staging of hepatitis C-related fibrosis: an updated metaanalysis. Hepatology 2011;53:726-736.

10. Jayanta Paul. Recent advances in non-invasive diagnosis and medical management of non-alcoholic fatty liver disease in adult. Egyptian Liver Journal 2020;10(37):1-18.

11. Abd El Rihim AY, Omar RF, Fathalah W, El Attar I, Hafez HA, Ibrahim W. Role of FibroScan ${ }^{\circledR}$ and APRI in detection of liver fi brosis: A systematic review and meta-analysis. Arab J Gastroenterol 2013;14(2):44-50.

12. Pathik P, Ravindra S, Ajay C, Prasad B, Jatin P, Prabha $\mathrm{S}$. Fibroscan versus simple noninvasive screening tools in predicting fibrosis in high-risk nonalcoholic fatty liver disease patients from Western India. Ann Gastroenterol 2015;28(2):281-286. PMID: 25830783; PMCID: PMC4367221. 